\title{
Review Article \\ Virosome Presents Multimodel Cancer Therapy without Viral Replication
}

\author{
Kotaro Saga and Yasufumi Kaneda \\ Division of Gene Therapy Science, Graduate School of Medicine, Osaka University, 2-2 Yamada-oka, Suita, Osaka 565-0871, Japan \\ Correspondence should be addressed to Yasufumi Kaneda; kaneday@gts.med.osaka-u.ac.jp
}

Received 8 October 2013; Accepted 31 October 2013

Academic Editor: Ryuichi Morishita

Copyright (C) 2013 K. Saga and Y. Kaneda. This is an open access article distributed under the Creative Commons Attribution License, which permits unrestricted use, distribution, and reproduction in any medium, provided the original work is properly cited.

\begin{abstract}
A virosome is an artificial envelope that includes viral surface proteins and lacks the ability to produce progeny virus. Virosomes are able to introduce an encapsulated macromolecule into the cytoplasm of cells using their viral envelope fusion ability. Moreover, virus-derived factors have an adjuvant effect for immune stimulation. Therefore, many virosomes have been utilized as drug delivery vectors and adjuvants for cancer therapy. This paper introduces the application of virosomes for cancer treatment. In Particular, we focus on virosomes derived from the influenza and Sendai viruses which have been widely used for cancer therapy. Influenza virosomes have been mainly applied as drug delivery vectors and adjuvants. By contrast, the Sendai virosomes have been mainly applied as anticancer immune activators and apoptosis inducers.
\end{abstract}

\section{Introduction}

Currently, general cancer therapies include surgery, chemotherapy, and radiation therapy, but all three have limitations. Applications of surgical and radiation therapy are limited to localized cancer. Chemotherapy is used for a wide range of cancers, including distant metastases, via the systemic administration of anti-cancer drugs; however, it also kills normal cells and induces severe side effects. Therefore, many groups are investigating ways to improve conventional treatments and to develop novel treatments for more effective cancer elimination with fewer side effects.

In recent years, much attention has been paid to cancer immunotherapy, which stimulates anti-cancer immunity, and several cancer immunotherapy systems (Provenge, Ipilimumab and anti-PD1 antibody) have been developed [15]. When anti-cancer immunity is systemically activated, it is expected that the primary cancer cells and distant metastases will be eliminated by immune cells. Various tumor-associated antigens (TAAs) have been identified [69], for example, HER2/nu, CEA, MAGE, and WT1. TAAs are expressed in cancer cells and are targeted by immune cells, especially cytotoxic T lymphocytes (CTL) [10-13]. Therefore, immunostimulation by TAAs can be applied to cancer immunotherapy. To activate anti-cancer immunity by TAAs, fragments of TAAs should be presented on antigenpresenting cells (APCs) by forming a complex with major histocompatibility complex class I (MHC-I) and II molecules [14]. Generally, cytoplasmic foreign proteins, such as viral proteins expressed in the cytoplasm during viral infection, complex with MHC-I and stimulate $\mathrm{CD} 8^{+} \mathrm{T}$ cells (CTLs) $[14,15]$. However, endocytosed foreign proteins also complex with MHC-II and stimulate $\mathrm{CD}^{+} \mathrm{T}$ cells $[14,16]$. Moreover, APCs have a cross-presentation system that presents endocytosed foreign proteins with MHC-I to activate CTLs [17]. Previous reports have shown that the administration of TAA alone does not induce an effective CTL response [18]. Therefore, it is believed that an endocytosed antigen is not sufficient for the activation of MHC-I-restricted CTLs, and, to activate an effective CTL response by TAAs, they should be introduced to the cytoplasm directly.

A new technology, gene therapy, has been developed and applied to cancer treatment. Various cancer gene therapy methods have been reported, such as adoptive immunotherapy using ex vivo gene transfer to immune cells [19], intratumoral injection of cytokine genes [20], suicide gene therapy using the herpes virus thymidine kinase gene [21], and intratumoral injection of the p53 gene [22]. To achieve 
high gene expression, viral vectors such as retrovirus and adenovirus vectors have been utilized. However, in general, cancer gene therapy has not had satisfactory therapeutic effects. Therefore, to enhance the cancer-cell-killing effect, viruses that replicate mainly in cancer cells have been used for treatment [23]. Various types of oncolytic viruses have been developed by isolating viruses with inherent tumor selectivity $[24,25]$ and by engineering recombinant viruses $[26,27]$. Furthermore, the combination of an oncolytic virus and gene therapy has been applied for cancer treatment, such as vaccinia virus including the GM-CSF gene [28]. Although these oncolytic viral treatments exhibited a strong therapeutic effect, safety might be a problem because the virus with an intact genome still exists in noncancerous cells [29].

An inactive virus that did not have the ability to amplify its progeny virus in host cells has also been used as a high-safety delivery vector for drugs and plasmids in cancer therapy. In particular, enveloped-virus-derived vectors have attracted attention because enveloped-vectordelivered molecules can escape endosomal degradation by direct introduction to the cytoplasm via membrane fusion [30]. A vector derived from an inactive enveloped virus is called a virosome, which is now an all-inclusive term for a reconstituted envelope that contains viral envelope proteins (Figure 1(a)) or viral envelope particles (Figure 1(b)) [31]. Several types of virosomes have been generated, for example, virosomes based on influenza virus [32], hepatitis $B$ virus [33], human immunodeficiency virus [34], Newcastle disease virus [35], and Sendai virus [36, 37]. In many studies, virosomes have been used as vectors for drug delivery, with the inclusion of various therapeutic molecules, such as DNA, RNA, proteins, and drugs [38, 39]. Moreover, virosomes function as adjuvants to induce the activation of the immune system [40]; therefore, many groups are studying virosomes as tools for cancer therapy.

In this review, we introduce the previous research on virosomes, especially virosomes derived from the influenza (influenza virosome) and Sendai viruses (Sendai virosome) for the use in cancer therapy. The influenza virosome has been applied mainly as a delivery vector for TAAs and TAAexpressing plasmids. Sendai virosomes have been used as anti-cancer immune activators and apoptosis inducers.

\section{Influenza Virosomes}

Influenza virus is an Orthomyxovirus that has a nucleocapsid with a segmented single-stranded RNA genome and is covered with a viral envelope $[41,42]$. Two types of membrane proteins, hemagglutinin (HA) and neuraminidase (NA), are present on the surface of the envelope. HA binds to sialic acid, which is its receptor, on the surface of host cells and is used for the adhesion of viral particles [43]. HA is responsible for membrane fusion of the viral envelope with the host cell membrane [44]. However, HA does not induce membrane fusion in neutral conditions, and it acquires its fusion activity through conformational change in acidic conditions $[45,46]$. Viral particles are taken into the endosomes of host cells by endocytosis after $\mathrm{HN}$-receptor binding, thereby exposing the particles to acidic conditions. Next, membrane fusion of the viral envelope with the endosomal membrane is induced by the conformational change of $\mathrm{HA}$, and the viral genome is induced into the cytoplasm of host cells.

An influenza virosome is an artificial liposome that includes influenza membrane proteins [31] and is prepared by reconstituting influenza virus surface proteins and phospholipids [47]. The influenza viral envelope is first collapsed to phospholipids by the treatment with detergent, and the nucleocapsid is eliminated from the mixture. Then, the influenza virosome, including surface proteins and virusderived phospholipids, is reconstituted from the mixture. An influenza virosome maintains its membrane fusion ability because it has HA on its surface [48]. Therefore, it works as a delivery vector to introduce macromolecules into the cytoplasm by including them in the virosome [38, 49]. Influenza virosomes have powerful immunogenicity. Vaccination with influenza virosomes induces protective levels of influenzaspecific antibodies [50], and an influenza virosome is already licensed as an influenza vaccine [51]. Influenza virosomes also exhibit an adjuvant effect when they are coadministered with other antigens [52-54]; therefore, many groups have studied the application of influenza virosomes in the activation of antitumor immunity.

2.1. CTL Activation by Plasmid DNA Encapsulation in Influenza Virosomes. Correale et al. reported that TAAspecific CTLs were induced by the administration of an influenza virosome containing TAA plasmids in mice [55]. In this study, a plasmid expressing parathyroid hormone-related peptide (PTH-rP), which is a TAA expressed in prostate and spinocellular lung carcinomas, was included in an influenza virosome, which was administered intranasally. As a result, PTH-rP-specific CTL activity was significantly induced in mice, and this activity was also shown in human PBMCs activated by human DCs treated with the PTH-rP virosome. In addition, Cusi et al. demonstrated that TAA-specific CTLs were enhanced by the stimulation with an influenza virosome containing a CD40L-expressing plasmid [56]. CD40L binds to $\mathrm{CD} 40$ on APCs and upregulates the expression of its costimulatory molecules, B7.1 and B7.2, in the cells, which are important factors for the activation and amplification of naïve $\mathrm{T}$ cells $[57,58]$. In this study, plasmids expressing carcinoembryonic antigen (CEA), which is a marker of colon cancer, and CD40L were encapsulated in influenza virosomes, and these virosomes were administered intranasally. Coadministration of CEA- and CD40L-virosomes resulted in a CEA-specific CTL response that was stronger than that in the CEA-virosome alone, by upregulating B7.1 and B7.2 expression on APCs.

2.2. CTL Activation by Peptide Encapsulation in Influenza Virosomes. Antigen presentation of TAAs by APCs is important for the activation of anti-cancer immunity. To activate CTLs, TAAs should be presented with MHC-I, which complexes with cytoplasmic antigens. Therefore, TAAs should be introduced to the cytoplasm for the effective activation 


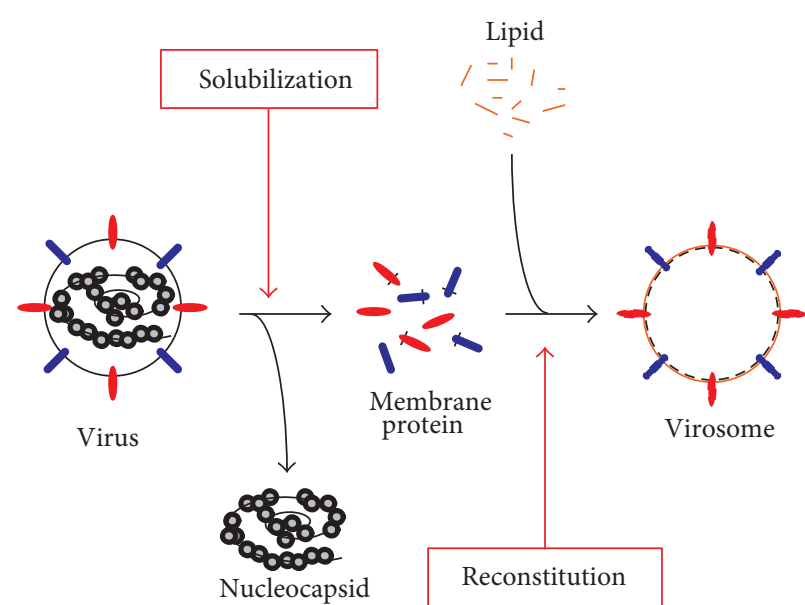

(a)

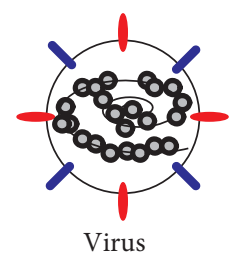

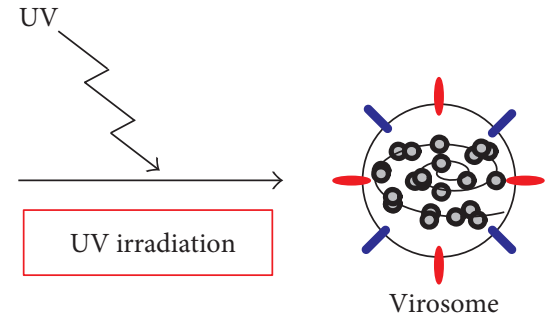

(b)

FIGURE 1: Concept of virosomes. (a) Reconstituted envelope containing viral envelope proteins. Viral membrane proteins are solubilized from viral particles, and artificial envelope is reconstituted with the viral proteins and exogenous lipids. (b) Viral envelope particles. Virus is inactivated with UV irradiation leading the fragmentation of viral genome.

of CTLs. Bungener et al. demonstrated influenza virosomemediated OVA delivery to DCs [59] and that the delivery leads to OVA presentation on MHC-I and -II. Fusioninactive virosomes presented OVA on MHC-II but not on -I. Therefore, it is suggested that influenza virosomes introduce encapsulated TAAs to the cytoplasm through membrane fusion and that TAA introduction is needed for the presentation of TAAs on MHC-I. Angel et al. reported influenza virosome-mediated delivery of TAAs to DCs [60]. The authors encapsulated the Melan-A peptide, which is a TAA from melanoma, in an influenza virosome and introduced the Melan-A peptide into plasmacytoid DCs (PDCs). Melan-A-containing, virosome-treated PDCs activated CD8 $\mathrm{T}$ cells more effectively than did free Melan-A peptide-pulsed PDCs. In addition, Correale et al. reported that PTH-rP-derived peptide (PTR)-4encapsulated influenza virosomes significantly suppressed tumor growth [61]. In this study, PTR-4/virosome treatment effectively activated CTL activity, and the treatment inhibited the angiogenesis of tumors. The findings therefore suggest a new function of influenza virosomes in cancer therapy.

2.3. Modification of the Influenza Virus. To make influenza virosome-mediated cancer therapy more effective, modifications of the influenza virosome have been attempted. HA has an important function in influenza virosome-mediated delivery and immunostimulation. However, the HA receptor is ubiquitously expressed on nearly all cells. Therefore, the influenza virosome does not have affinity for specific cells. Mastrobattista et al. generated an influenza virosome that could target ovarian carcinoma (OVCAR-3) in vitro [62]. They coated influenza virosomes with polyethylene glycol (PEG) to inhibit HA-mediated binding, and then Fab' fragments of antiepithelial glycoprotein-2 (EGP-2) antibody
(323/A3) were conjugated to the PEG on the virosomes. 323/A3-PEG-coated influenza virosomes exhibited low HAmediated binding to sialic acid because of the PEG coating and gained specific binding for EGP-2-expressing ovarian cancer cells by 323/A3 conjugation. As a result, although the binding function of HA was depleted, the 323/A3-PEG virosomes were able to fuse with OVCAR-3 membranes. Because HA induced membrane fusion without binding to its receptor [63], it is thought that the 323/A3-PEG virosomes maintained their membrane fusion ability. Waelti et al. used the same strategy to demonstrate targeted delivery of doxorubicin (Doxo) to HER-2/neu-overexpressing breast cancer cells in vivo [64]. In this study, influenza virosomes were coated with anti-Neu mAb Fab' (7.16.4)-conjugated PEG (7.16.4/PEG), and Doxo was encapsulated in the 7.16.4/PEG-virosomes. Intravenous administration of Doxo-containing 7.16.4/PEGvirosomes significantly inhibited subcutaneous $\mathrm{Neu}+$, but not Neu-, breast cancer. Jamali et al. recently reported the enhancement of the efficacy of influenza virosome-mediated delivery in vitro by reconstituting the virosome with cationic lipids [65].

As described above, influenza virosomes are useful for the cancer therapy. Recently, phase I clinical trial of influenza virosomes was carried out for the patients with metastatic breast cancer (MBC) [66]. In this trial, MBC patients were intramuscularly administrated influenza virosomes including three individual peptides of the extracellular domain of Her-2/neu protein. The trial tested the safety and Her-2/neuspecific immune responses. As a result, specific antibodies against naïve Her-2/neu protein were detected in serum. IL-2 production was significantly increased and Treg population was significantly decreased in PBMC. Although local erythema at the infection site has appeared in four patients, other serious side effects were not detected. Therefore, there is a possibility that influenza virosomes are used for future cancer therapy. 


\section{Sendai Virosomes}

Sendai virus (hemagglutinating virus of Japan; HVJ) is a paramyxovirus that has a nucleocapsid with a single-stranded RNA genome and is covered with a viral envelope [67]. Two types of glycoproteins, hemagglutinin-neuraminidase $(\mathrm{HN})$ and fusion protein $(\mathrm{F})$, are present on the surface of the viral envelope [68]. HN enables the viral particle to adhere to the host-cell surface by binding to sialic acid [69], and then $\mathrm{F}$ induces membrane fusion of the viral envelope with the host-cell membrane [70]. F fuses these membranes under neutral conditions [71]; therefore, HVJ particles do not require uptake into the endosome for membrane fusion.

Previously, HVJ-liposomes were generated via reconstitution from HVJ surface proteins and phospholipids, similar to the influenza virosome [36]. Because HVJ-liposomes have membrane fusion ability, they have been used as a vector for DNA delivery [72]. However, because the membrane fusion efficiency of HVJ-liposomes is not high (approximately 2\% of native $\mathrm{HVJ}$ ) [73], an increase in the fusion activity of the vector is needed.

Kaneda et al. generated a new type of Sendai virosomes called HVJ-envelope (HVJ-E) [37]. HVJ-E is an inactivated HVJ particle that has been irradiated by UV light. The viral RNA genome is cleaved into many fragments; therefore, HVJ-E does not have the ability to produce progeny virus in infected cells. However, HVJ-E maintains its membrane fusion ability, which is dramatically higher than that of HVJliposomes [37]. HVJ-E has been used as a vector for plasmid DNA delivery to various cells and tissues [74-76]. In addition, plasmid DNA, anti-cancer drugs, and siRNAs have been delivered by HVJ-E, and there have been reports of cancer therapy using HVJ-E-mediated drug delivery [77, 78].

Cancer suppression by viral infection has also been reported [79]. Since that study, various viruses have been used for cancer therapy, and, in particular, the development of oncolytic viruses has attracted attention [80-83]. Oncolytic viruses function by inducing the lysis of cancer cells by infection [84]. Because the oncolytic activity is decreased by UV irradiation, it has been suggested that the viral amplification in cancer cells is responsible for oncolysis [85]. However, it is also possible that the virus's components contribute to the suppression of cancer. Recently, it was indicated that HVJ-E itself has an inhibitory effect against cancer growth $[86,87]$, and it was revealed that the viral components, in the absence of viral amplification, contribute to the anti-cancer effects. Since then, the HVJ-E-mediated anti-cancer effect has been studied.

3.1. HVJ-E for the Activation of Anticancer Immunity. Kurooka and Kaneda demonstrated that the intratumoral administration of HVJ-E dramatically eradicated intradermal cancer (Figure 2) [86]. They found that HVJ-E stimulated DCs to release various types of cytokines, such as interferon (IFN)- $\alpha$, and $-\beta$, tumor-necrosis factor (TNF)- $\alpha$ and interleukin (IL)-6, and that IL-6 inhibited the proliferation of regulatory $\mathrm{T}$ cells (Tregs). Tregs negatively control effector $\mathrm{T}$ cells $[88,89]$ and interfere with the activation of anti-cancer immunity [90]. Therefore, HVJ-E-mediated eradication

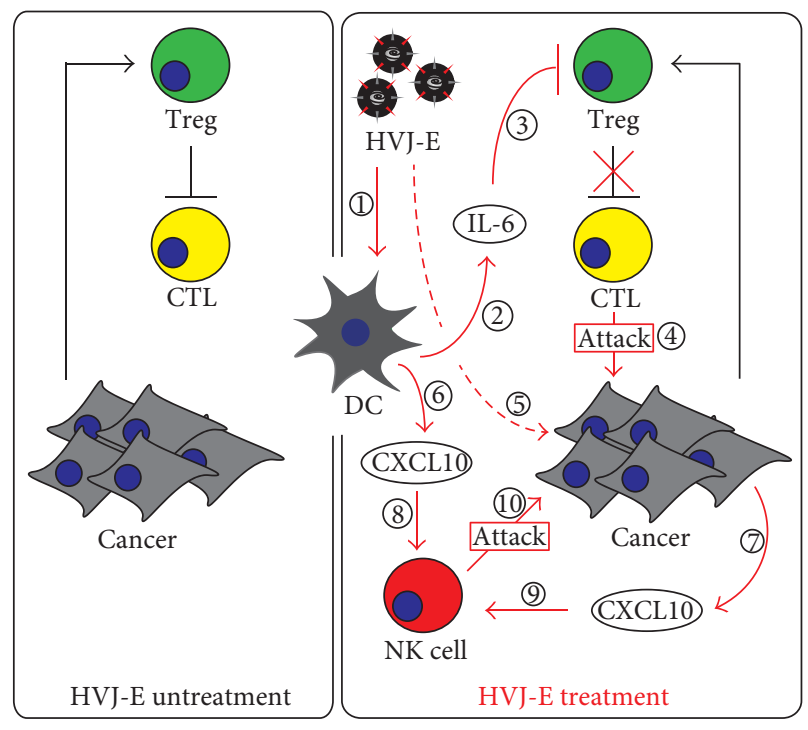

FIgURE 2: HVJ-E-mediated stimulation of anticancer immunity. Various modes of stimulations of the immune system that are induced by HVJ-E treatment. Black lines indicate the original reactions in cancer. Red lines indicate the reactions induced by HVJ-E treatment. CTL Activation. 1: HVJ-E stimulates dendritic cells (DCs). 2: DC secretes IL-6. 3: IL-6 suppresses regulatory T cell (Treg) function, which inhibits cytotoxic T cell (CTL) activity. 4: CTLs attack cancer cells. NK Cell Activation. 1, 5: HVJ-E stimulates DCs and cancer cells. 6, 7: DCs and cancer cells secrete CXCL10. 8, 9: CXCL10 attracts natural killer (NK) cells to cancer cells. 10: NK cells effectively attack the cancer cells.

of cancer results from the activation of anti-cancer immunity by IL-6-mediated suppression of Tregs. It is known that RNA viruses stimulate DCs via the recognition of the viral RNA genome by Toll-like receptor (TLR)-7 and - 8 and Rig-I [91-93]. However, Suzuki et al. showed that the sugar chain of the $\mathrm{F}$ protein is important for HVJ-E-mediated, DC activation of IL-6 secretion [94]. Therefore, they suggested that DCs possess an unknown receptor for $\mathrm{F}$ that is involved in maturation.

In addition, HVJ-E suppressed tumor growth in the intradermal renal carcinoma SCID mouse model, in spite of their deficient T and B cells [95], which suggests that HVJ-E undergoes another stimulation mechanism that activates anti-cancer immunity. Fujihara et al. indicated that the anticancer activity of NK cells was led by the intratumoral administration of HVJ-E into the intradermal renal carcinoma SCID mouse model (Figure 1) [95]. In addition, HVJ-E directly stimulated cancer cells and induced their secretion of CXCL10. CXCL10 is a chemokine for monocytes/macrophages, T cells, NK cells, and DCs; therefore, it is suggested that active NK cells were attracted to the tumor by CXCL10.

Taken together, these reports demonstrate that HVJ-E is a powerful activator of anti-cancer immunity.

3.2. Direct Cancer Killing Activity of HVJ-E. Recently, it was reported that $\mathrm{HVJ}-\mathrm{E}$ has a direct killing effect against cancer 


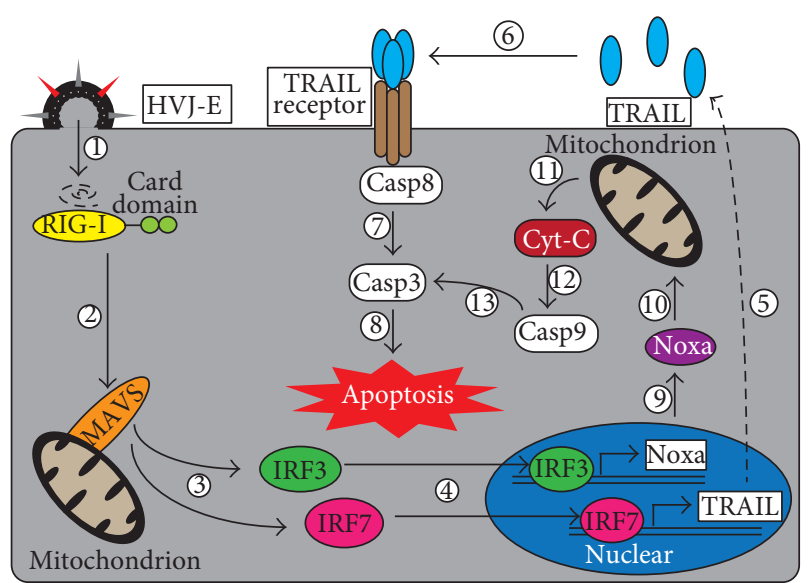

FIGURE 3: HVJ-E-mediated apoptosis pathway in cancer cells. HVJE-mediated signal transduction in cancer cells. 1: RNA fragments derived from the HVJ genome are introduced into the cytoplasm by membrane fusion, and RIG-I recognizes these RNAs. 2: RIG-I conveys the signal to the mitochondrial antiviral signaling adaptor (MAVS). 3: MAVS activates IRF-7 and -3. 4: activated IRF-7 and -3 localize to the nucleus. 5, 9: IRF-7 and -3 induce the expression of TRAIL and Noxa. 6: expressed TRAILs are recognized by the TRAIL receptor, and TRAIL receptors activate caspase-8 (Casp-8). 7: activated Casp-8 activates Casp-3. 8: activated Casp-3 induces apoptosis. 10, 11: Noxa induces the secretion of cytochrome-C (CytC) into the cytoplasm from the mitochondria. 12: Cyt- $\mathrm{C}$ activates Casp-9. 13: Casp-9 activates Casp-3.

cells (Figure 3). Kawaguchi et al. showed that the viability of two castration-resistant human prostate cancer cell lines (PC3 and DU145) was remarkably decreased by the treatment with HVJ-E in vitro [96]. HVJ-E-treated PC3 cells exhibited some apoptotic phenotypes, namely, increases in the number of TUNEL-stained cells and in the expression levels of caspase- 3 and caspase- 8 . However, HVJ-E-mediated inhibition of cell viability was not observed in normal prostate epithelium (PNT2), suggesting that HVJ-E-mediated apoptosis is specifically induced in cancer cells. HVJ-E contains many fragments of its RNA genome, and these RNA fragments are introduced to the cytoplasm by the fusion of HVJ-E and the cell membrane. Matsushima-Miyagi et al. revealed that the viability of prostate cancer cells (PC3 and DU145), but not normal prostate epithelium (PNT1 and PNT2), was significantly decreased by viral RNA introduction (Figure 2) [87]. The RNA fragments were recognized by RIG-I in the cytoplasm, and the signal was transduced to MAVS [97]. HVJ-E-mediated cell growth inhibition of PC3 was suppressed by the knockdown of RIG-I and MAVS, indicating that the RIG-I/MAVS signaling pathway is important for this process. Moreover, HVJ-E treatment induced the expression of TRAIL and Noxa (known as apoptosis inducers $[98,99]$ ) in PC3 and DU145 cells, but not in PNT2 cells, via RIG-I/MAVS signaling. The fact that the knockdown of TRAIL and Noxa suppressed the HVJ-E sensitivity of PC3 and DU145, respectively, indicates that these apoptosis inducers are responsible for HVJ-E-induced cancer cell apoptosis. Furthermore, the knockdown of IRF7 and 3-transcription factors of TRAIL and Noxa, respectively $[100,101]$ - also suppressed the HVJ-E sensitivity of prostate cancer cells, suggesting that RIG-I/ MAVS signaling regulates the expression of TRAIL and Noxa via IRF7 and 3 in cancer cells. Matsushima-Miyagi et al. [87] elucidates the mechanism of HVJ-E-induced cancer cell apoptosis. However, it is still unknown why the expression of these apoptosis inducers is induced in cancer cells by HVJ-E stimulation.

3.3. Combination Therapy with HVJ-E and Modification of $H V J-E$. In attempts to enhance the strength and decrease the side effects of HVJ-E-mediated antitumor treatment, various combination therapies that include HVJ-E and modifications of HVJ-E have been used. Eg5 is an important factor in the early stages of mitosis [102] and its inhibition leads to mitotic arrest and results in apoptosis [103]. Matsuda et al. demonstrated that HVJ-E-mediated apoptosis in human glioblastoma cell lines (A-172, T98G and U-118MG) was effectively enhanced by the encapsulation of siRNAs against Eg5 in HVJ-E in vitro and in vivo [104]. The authors also observed that HVJ-E-mediated anti-cancer immunity was enhanced by the encapsulation of the IL-2 plasmid and that the astrocytoma cell line (RSV-M) was effectively eradicated when using this method in vivo [105].

HVJ-E adheres to the cell surface via $\mathrm{HN}$ binding to sialic acid (e.g., GDla and SPG) [106]. Therefore, cancer cells with mild expression of these sialic acids exhibit low sensitivity to HVJ-E-mediated apoptosis because of their weak affinity for HVJ-E. To induce HVJ-E-mediated apoptosis in less sensitive cancer cells, Nomura et al. used the combination therapy of HVJ-E and 13-cis retinoic acid (13cRA) against human neuroblastoma cells (NB1), which are less sensitive to HVJ-E [107]. NB1 cells barely express GDla and SPG and exhibit low sensitivity to HVJ-E-mediated apoptosis. 13cRA treatment induced the expression of GDla in NB1 cells, and the HVJ-E sensitivity of NB1 cells was increased in vitro. Moreover, NB1 tumor volume in mice was significantly decreased and their survival rate was increased by the combination of HVJ-E and 13cRA in vivo.

Improvements to HVJ-E were made to enhance its performance. Sialic acids, such as GDla and SPG, to which HN bind, are ubiquitously expressed in nearly all cells, and they are highly expressed in red blood cells. Therefore, HVJ-E does not have an affinity for a specific cell type, and it induces hemagglutination by intravenous administration. For the systemic administration of HVJ-E to treat cancer effectively, it must have high affinity for cancer cells and low affinity for sialic acids. Transferrin (Tf) is a protein in blood plasma that is responsible for ferric ion delivery, and the Tf receptor is highly expressed in various cancer cells. Shimbo et al. generated a cancer-targeting HVJ-E using Tf [108]. The HN on HVJ-E was depleted by siRNA [109], and Tf was presented on the surface of HVJ-E via the expression of a Tf/F recombinant fusion protein on HVJ-E. Tf-presented HVJ-E (Tf-HVJ-E) exhibited affinity for the human uterocervical cancer cell (Hela) line, which expressed the Tf receptor, and Tf-HVJ-E accumulated at tumor masses in mice after their systemic administration. 
In addition, HVJ-E-mediated antitumor immunity was enhanced by HVJ-E modification. HVJ-E activates anticancer immunity; however, HVJ-E does not directly induce IFN- $\gamma$ secretion. IFN- $\gamma$ is an important factor for various anti-cancer activities [110], and IL-12 is a robust inducer of IFN- $\gamma$ from immune cells $[111,112]$. Saga et al. revealed that HVJ-E dramatically enhanced IL-12 activity for IFN- $\gamma$ secretion from splenocytes; however, HVJ-E alone did not induce IFN- $\gamma$ secretion [113]. The authors generated IL12-conjugated- and HN-depleted HVJ-E (IL-12-HVJ-E) to enhance HVJ-E-mediated anti-cancer immunity. IL-12-HVJ$\mathrm{E}$ induced secretion of IFN- $\gamma$ from splenocytes in vitro. In addition, upon intratumoral injection, scIL12-HVJ-E activated antitumor immunity against mouse malignant melanomas (F10 melanoma) and suppressed tumor growth more effectively than the wild-type (wt) HVJ-E. Furthermore, upon intravenous injection, IL-12-HVJ-E, but not wt-HVJ-E, was especially localized to the lungs, where it induced IFN$\gamma$ expression and reduced the lung metastatic foci of F10 melanomas.

As described above, HVJ-E has the ability to induce anticancer effects in several types of cancers. Now, clinical trials of HVJ-E are ongoing to test its safety and anti-cancer immunity against melanoma and prostate cancer. Moreover, there is a possibility that the combination therapy of HVJ-E and other immune therapies, such as CTLA- 4 antibody, exhibits a more effective activation of antitumor immunity, and it will be performed in the near future.

\section{Conclusion}

We have documented the utility of virosomes for cancer treatment. However, we believe that no omnipotent therapeutic technologies are currently available to completely eradicate various types of cancers. Cancers are heterogeneous and can transform themselves to be resistant to the treatment that they have received and to escape from the environment of cancer treatment [114]. In this scientific research field, it is absolutely necessary to identify the genes that direct tumorigenesis. However, in the clinical field, it is very important to prepare cancer treatments using a variety of therapeutic principles. Clinicians should provide cancer patients with the appropriate therapeutic tools according to the patient's condition. Thus, from a practical standpoint, virosome-mediated cancer therapy may have an important role in cancer treatment.

\section{Conflict of Interests}

The authors declare that there is no conflict of interests.

\section{References}

[1] P. A. Burch, G. A. Croghan, D. A. Gastineau et al., "Immunotherapy (APC8015, provenge) targeting prostatic acid phosphatase can induce durable remission of metastatic androgenindependent prostate cancer: a phase 2 trial," Prostate, vol. 60, no. 3, pp. 197-204, 2004.
[2] M. A. Cheever and C. S. Higano, "PROVENGE (sipuleucel-T) in prostate cancer: the first FDA-approved therapeutic cancer vaccine," Clinical Cancer Research, vol. 17, no. 11, pp. 3520-3526, 2011.

[3] F. S. Hodi, S. J. O’Day, D. F. McDermott et al., "Improved survival with ipilimumab in patients with metastatic melanoma," The New England Journal of Medicine, vol. 363, no. 8, pp. 711723, 2010.

[4] P. A. Prieto, J. C. Yang, R. M. Sherry et al., "CTLA-4 blockade with ipilimumab: long-term follow-up of 177 patients with metastatic melanoma," Clinical Cancer Research, vol. 18, no. 7, pp. 2039-2047, 2012.

[5] S. L. Topalian, F. S. Hodi, J. R. Brahmer et al., "Safety, activity, and immune correlates of anti-PD-1 antibody in cancer," The New England Journal of Medicine, vol. 366, no. 26, pp. 24432454, 2012.

[6] R.-F. Wang, "Human tumor antigens: implications for cancer vaccine development," Journal of Molecular Medicine, vol. 77, no. 9, pp. 640-655, 1999.

[7] K. M. Call, T. Glaser, C. Y. Ito et al., "Isolation and characterization of a zinc finger polypeptide gene at the human chromosome 11 Wilms' tumor locus," Cell, vol. 60, no. 3, pp. 509-520, 1990.

[8] M. Gessler, A. Poustka, W. Cavenee, R. L. Neve, S. H. Orkin, and G. A. P. Bruns, "Homozygous deletion in Wilms tumours of a zinc-finger gene identified by chromosome jumping," Nature, vol. 343, no. 6260, pp. 774-778, 1990.

[9] P. van der Bruggen, C. Traversari, P. Chomez et al., "A gene encoding an antigen recognized by cytolytic T lymphocytes on a human melanoma," Science, vol. 254, no. 5038, pp. 1643-1647, 1991.

[10] M. Peiper, P. S. Goedegebuure, J. R. Izbicki, and T. J. Eberlein, "Pancreatic cancer associated ascites-derived CTL recognize a nine-amino-acid peptide GP2 derived from HER2/neu," Anticancer Research, vol. 19, no. 4, pp. 2471-2475, 1999.

[11] M. Peiper, P. S. Goedegebuure, D. C. Linehan, E. Ganguly, C. C. Douville, and T. J. Eberlein, "The HER2/neu-derived peptide p654-662 is a tumor-associated antigen in human pancreatic cancer recognized by cytotoxic T lymphocytes," European Journal of Immunology, vol. 27, no. 5, pp. 1115-1123, 1997.

[12] P. Holliger, O. Manzke, M. Span et al., "Carcinoembryonic antigen (CEA)-specific T-cell activation in colon carcinoma induced by anti-CD3 $\times$ anti-CEA bispecific diabodies and B7×anti- CEA bispecific fusion proteins," Cancer Research, vol. 59, no. 12, pp. 2909-2916, 1999.

[13] Y. Oka, O. A. Elisseeva, A. Tsuboi et al., "Human cytotoxic T-lymphocyte responses specific for peptides of the wild-type Wilms' tumor gene (WT1) product," Immunogenetics, vol. 51, no. 2, pp. 99-107, 2000.

[14] R. N. Germain, "MHC-dependent antigen processing and peptide presentation: providing ligands for $\mathrm{T}$ lymphocyte activation," Cell, vol. 76, no. 2, pp. 287-299, 1994.

[15] M. Jondal, R. Schirmbeck, and J. Reimann, "MHC class Irestricted CTL responses to exogenous antigens," Immunity, vol. 5, no. 4, pp. 295-302, 1996.

[16] I. Mellman, S. J. Turley, and R. M. Steinman, "Antigen processing for amateurs and professionals," Trends in Cell Biology, vol. 8, no. 6, pp. 231-237, 1998.

[17] P. Cresswell, A. L. Ackerman, A. Giodini, D. R. Peaper, and P. A. Wearsch, "Mechanisms of MHC class I-restricted antigen processing and cross-presentation," Immunological Reviews, vol. 207, pp. 145-157, 2005. 
[18] M. Marchand, N. van Baren, P. Weynants et al., "Tumor regressions observed in patients with metastatic melanoma treated with an antigenic peptide encoded by gene MAGE-3 and presented by HLA-A1," British Journal of Cancer, vol. 80, pp. 219-230, 1999.

[19] M. H. Kershaw, J. A. Westwood, and P. K. Darcy, "Geneengineered T cells for cancer therapy," Nature Reviews Cancer, vol. 13, pp. 525-541, 2013.

[20] C.-Y. Li, Q. Huang, and H.-F. Kung, "Cytokine and immunogene therapy for solid tumors," Cellular \& Molecular Immunology, vol. 2, no. 2, pp. 81-91, 2005.

[21] C. Fillat, M. Carrió, A. Cascante, and B. Sangro, "Suicide gene therapy mediated by the Herpes Simplex virus thymidine kinase gene/Ganciclovir system: fifteen years of application," Current Gene Therapy, vol. 3, no. 1, pp. 13-26, 2003.

[22] J. A. Roth, "Adenovirus p53 gene therapy," Expert Opinion on Biological Therapy, vol. 6, no. 1, pp. 55-61, 2006.

[23] D. Kirn, R. L. Martuza, and J. Zwiebel, "Replication-selective virotherapy for cancer: biological principles, risk management and future directions," Nature Medicine, vol. 7, no. 7, pp. 781-787, 2001.

[24] R. M. Lorence, P. A. Rood, and K. W. Kelley, "Newcastle disease virus as an antineoplastic agent: induction of tumor necrosis factor- $\alpha$ and augmentation of its cytotoxicity," Journal of the National Cancer Institute, vol. 80, no. 16, pp. 1305-1312, 1988.

[25] M. C. Coffey, J. E. Strong, P. A. Forsyth, and P. W. K. Lee, "Reovirus therapy of tumors with activated Ras pathway," Science, vol. 282, no. 5392, pp. 1332-1334, 1998.

[26] R. L. Martuza, A. Malick, J. M. Markert, K. L. Ruffner, and D. M. Coen, "Experimental therapy of human glioma by means of a genetically engineered virus mutant," Science, vol. 252, no. 5007, pp. 854-856, 1991.

[27] J. R. Bischoff, D. H. Kirn, A. Williams et al., "An adenovirus mutant that replicates selectively in p53-deficient human tumor cells," Science, vol. 274, no. 5286, pp. 373-376, 1996.

[28] M. J. Mastrangelo, H. C. Maguire Jr., L. C. Eisenlohr et al., "Intratumoral recombinant GM-CSF-encoding virus as gene therapy in patients with cutaneous melanoma," Cancer Gene Therapy, vol. 6, no. 5, pp. 409-422, 1999.

[29] S. H. Thorne, T.-H. H. Hwang, W. E. O'Gorman et al., "Rational strain selection and engineering creates a broad-spectrum, systemically effective oncolytic poxvirus, JX-963," The Journal of Clinical Investigation, vol. 117, no. 11, pp. 3350-3358, 2007.

[30] L. J. Earp, S. E. Delos, H. E. Park, and J. M. White, "The many mechanisms of viral membrane fusion proteins," in Membrane Trafficking in Viral Replication, D. M. Marsh, Ed., pp. 25-66, Springer, Berlin, Germany, 2005.

[31] J. Almeida, D. C. Edwards, C. Brand, and T. Heath, "Formation of virosomes from influenza subunits and liposomes," The Lancet, vol. 306, pp. 899-901, 1975.

[32] R. Zurbriggen, "Immunostimulating reconstituted influenza virosomes," Vaccine, vol. 21, no. 9-10, pp. 921-924, 2003.

[33] T. Yamada, Y. Iwasaki, H. Tada et al., "Nanoparticles for the delivery of genes and drugs to human hepatocytes," Nature Biotechnology, vol. 21, no. 8, pp. 885-890, 2003.

[34] B. Cornet, M. Vandenbranden, J. Cogniaux, L. Giurgea, D. Dekegel, and J. M. Ruysschaert, "Virosomes reconstituted from human immunodeficiency virus proteins and lipids," Biochemical and Biophysical Research Communications, vol. 167, no. 1, pp. 222-231, 1990.
[35] D. R. Kapczynski and T. M. Tumpey, "Development of a virosome vaccine for Newcastle disease virus," Avian Diseases, vol. 47, no. 3, pp. 578-587, 2003.

[36] T. Uchida, J. Kim, M. Yamaizumi, Y. Miyake, and Y. Okada, "Reconstitution of lipid vesicles associated with HVJ (Sendai virus) spikes. Purification and some properties of vesicles containing nontoxic fragment A of diphtheria toxin," Journal of Cell Biology, vol. 80, no. 1, pp. 10-20, 1979.

[37] Y. Kaneda, T. Nakajima, T. Nishikawa et al., "Hemagglutinating virus of Japan (HVJ) envelope vector as a versatile gene delivery system," Molecular Therapy, vol. 6, no. 2, pp. 219-226, 2002.

[38] D. Felnerova, J.-F. Viret, R. Glück, and C. Moser, "Liposomes and virosomes as delivery systems for antigens, nucleic acids and drugs," Current Opinion in Biotechnology, vol. 15, no. 6, pp. 518-529, 2004.

[39] P. E. Lund, R. C. Hunt, M. M. Gottesman, and C. KimchiSarfaty, "Pseudovirions as vehicles for the delivery of siRNA," Pharmaceutical Research, vol. 27, no. 3, pp. 400-420, 2010.

[40] C. Moser, I. C. Metcalfe, and J.-F. Viret, "Virosomal adjuvanted antigen delivery systems," Expert Review of Vaccines, vol. 2, no. 2, pp. 189-196, 2003.

[41] D. L. Suarez and S. Schultz-Cherry, "Immunology of avian influenza virus: a review," Developmental and Comparative Immunology, vol. 24, no. 2-3, pp. 269-283, 2000.

[42] T. Noda and Y. Kawaoka, "Structure of influenza virus ribonucleoprotein complexes and their packaging into virions," Reviews in Medical Virology, vol. 20, no. 6, pp. 380-391, 2010.

[43] J. J. Skehel and D. C. Wiley, "Receptor binding and membrane fusion in virus entry: the influenza hemagglutinin," Annual Review of Biochemistry, vol. 69, pp. 531-569, 2000.

[44] S. Fukuyama and Y. Kawaoka, "The pathogenesis of influenza virus infections: the contributions of virus and host factors," Current Opinion in Immunology, vol. 23, no. 4, pp. 481-486, 2011.

[45] K. J. Cross, L. M. Burleigh, and D. A. Steinhauer, "Mechanisms of cell entry by influenza virus," Expert Reviews in Molecular Medicine, vol. 3, pp. 1-18, 2001.

[46] A. Yoshimura, K. Kuroda, K. Kawasaki, S. Yamashina, T. Maeda, and S. I. Ohnishi, "Infectious cell entry mechanism of influenza virus," Journal of Virology, vol. 43, no. 1, pp. 284-293, 1982.

[47] R. Bron, A. Ortiz, J. Dijkstra, T. Stegmann, and J. Wilschut, "Preparation, properties, and applications of reconstituted influenza virus envelopes (virosomes)," in Methods in Enzymology, N. Duzgunees, Ed., pp. 313-331, Academic Press, San Diego, Calif, USA, 1993.

[48] T. Stegmann, H. W. Morselt, F. P. Booy, J. F. van Breemen, G. Scherphof, and J. Wilschut, "Functional reconstitution of influenza virus envelopes," The EMBO Journal, vol. 6, no. 9, pp. 2651-2659, 1987.

[49] R. Bron, A. Ortiz, and J. Wilschut, "Cellular cytoplasmic delivery of a polypeptide toxin by reconstituted influenza virus envelopes (virosomes)," Biochemistry, vol. 33, no. 31, pp. 91109117, 1994.

[50] R. Glück, R. Mischler, B. Finkel, J. U. Que, B. Scarpa, and S. J. Cryz Jr., "Immunogenicity of new virosome influenza vaccine in elderly people," The Lancet, vol. 344, no. 8916, pp. 160-163, 1994.

[51] R. Mischler and I. C. Metcalfe, "Inflexal V a trivalent virosome subunit influenza vaccine: production," Vaccine, vol. 20, no. 5, pp. B17-B23, 2002.

[52] R. Zurbriggen and R. Glück, "Immunogenicity of IRIV- versus alum-adjuvanted diphtheria and tetanus toxoid vaccines in 
influenza primed mice," Vaccine, vol. 17, no. 11-12, pp. 1301-1305, 1999.

[53] R. Zurbriggen, I. Novak-Hofer, A. Seelig, and R. Glück, "IRIVadjuvanted hepatitis A vaccine: in vivo absorption and biophysical characterization," Progress in Lipid Research, vol. 39, no. 1, pp. 3-18, 2000.

[54] F. Pöltl-Frank, R. Zurbriggen, A. Helg et al., "Use of reconstituted influenza virus virosomes as an immunopotentiating delivery system for a peptide-based vaccine," Clinical and Experimental Immunology, vol. 117, no. 3, pp. 496-503, 1999.

[55] P. Correale, M. G. Cusi, M. Sabatino et al., "Tumour-associated antigen (TAA)-specific cytotoxic T cell (CTL) response in vitro and in a mouse model, induced by TAA-plasmids delivered by influenza virosomes," European Journal of Cancer, vol. 37, no. 16, pp. 2097-2103, 2001.

[56] M. G. Cusi, M. T. del Vecchio, C. Terrosi et al., "Immunereconstituted influenza virosome containing CD40L gene enhances the immunological and protective activity of a carcinoembryonic antigen anticancer vaccine," Journal of Immunology, vol. 174, no. 11, pp. 7210-7216, 2005.

[57] Y. Yang and J. M. Wilson, "CD40 ligand-dependent T cell activation: requirement of B7-CD28 signaling through CD40," Science, vol. 273, no. 5283, pp. 1862-1864, 1996.

[58] S. P. Schoenberger, R. E. M. Toes, E. I. H. van Dervoort, R. Offringa, and C. J. M. Melief, "T-cell help for cytotoxic T lymphocytes is mediated by CD40-CD4OL interactions," Nature, vol. 393, no. 6684 , pp. 480-483, 1998.

[59] L. Bungener, K. Serre, L. Bijl et al., "Virosome-mediated delivery of protein antigens to dendritic cells," Vaccine, vol. 20, no. 17-18, pp. 2287-2295, 2002.

[60] J. Angel, L. Chaperot, J.-P. Molens et al., "Virosome-mediated delivery of tumor antigen to plasmacytoid dendritic cells," Vaccine, vol. 25, no. 19, pp. 3913-3921, 2007.

[61] P. Correale, M. T. del Vecchio, T. Renieri et al., "Anti-angiogenetic effects of immune-reconstituted influenza virosomes assembled with parathyroid hormone-related protein derived peptide vaccine," Cancer Letters, vol. 263, no. 2, pp. 291-301, 2008.

[62] E. Mastrobattista, P. Schoen, J. Wilschut, D. J. A. Crommelin, and G. Storm, "Targeting influenza virosomes to ovarian carcinoma cells," FEBS Letters, vol. 509, no. 1, pp. 71-76, 2001.

[63] P. Schoen, L. Leserman, and J. Wilschut, "Fusion of reconstituted influenza virus envelopes with liposomes mediated by streptavidin/biotin interactions," FEBS Letters, vol. 390, no. 3, pp. 315-318, 1996.

[64] E. Waelti, N. Wegmann, R. Schwaninger et al., "Targeting HER2/neu with antirat neu virosomes for cancer therapy," Cancer Research, vol. 62, no. 2, pp. 437-444, 2002.

[65] A. Jamali, M. Holtrop, A. de Haan et al., "Cationic influenza virosomes as an adjuvanted delivery system for CTL induction by DNA vaccination," Immunology Letters, vol. 148, no. 1, pp. 77-82, 2012.

[66] U. Wiedermann, C. Wiltschke, J. Jasinska et al., "A virosomal formulated Her-2/neu multi-peptide vaccine induces Her2/neu-specific immune responses in patients with metastatic breast cancer: a phase I study," Breast Cancer Research and Treatment, vol. 119, no. 3, pp. 673-683, 2010.

[67] J. Curran and D. Kolakofsky, "Replication of paramyxoviruses," Advances in Virus Research, vol. 54, pp. 403-422, 1999.

[68] Y. Okada, "Sendai virus-induced cell fusion," in Methods in Enzymology, N. Duzgunes, Ed., pp. 18-41, Academic Press, San Diego, Calif, USA, 1993.
[69] T. Takimoto, G. L. Taylor, H. C. Connaris, S. J. Crennell, and A. Portner, "Role of the hemagglutinin-neuraminidase protein in the mechanism of paramyxovirus-cell membrane fusion," Journal of Virology, vol. 76, no. 24, pp. 13028-13033, 2002.

[70] A. Asano and K. Asano, "Viral proteins in cell fusion," Tokai Journal of Experimental and Clinical Medicine, vol. 7, supplement, pp. 193-196, 1982.

[71] A. M. Haywood and B. P. Boyer, "Sendai virus membrane fusion: time course and effect of temperature, $\mathrm{pH}$, calcium, and receptor concentration," Biochemistry, vol. 21, no. 24, pp. 6041$6046,1982$.

[72] Y. Kaneda, Y. Saeki, and R. Morishita, "Gene therapy using HVJliposomes: the best of both worlds?" Molecular Medicine Today, vol. 5, no. 7, pp. 298-303, 1999.

[73] Y. Kaneda, "New vector innovation for drug delivery: development of fusigenic non-viral particles," Current Drug Targets, vol. 4, no. 8, pp. 599-602, 2003.

[74] M. Shimamura, R. Morishita, M. Endoh et al., "HVJ-envelope vector for gene transfer into central nervous system," Biochemical and Biophysical Research Communications, vol. 300, no. 2, pp. 464-471, 2003.

[75] K. Oshima, M. Shimamura, S. Mizuno et al., "Intrathecal injection of HVJ-E containing HGF gene to cerebrospinal fluid can prevent and ameliorate hearing impairment in rats," The FASEB Journal, vol. 18, no. 1, pp. 212-214, 2004.

[76] Y. D. Kim, K.-G. Park, R. Morishita et al., "Liver-directed gene therapy of diabetic rats using an HVJ-E vector containing EBV plasmids expressing insulin and GLUT 2 transporter," Gene Therapy, vol. 13, no. 3, pp. 216-224, 2006.

[77] M. Ito, S. Yamamoto, K. Nimura, K. Hiraoka, K. Tamai, and Y. Kaneda, "Rad51 siRNA delivered by HVJ envelope vector enhances the anti-cancer effect of cisplatin," Journal of Gene Medicine, vol. 7, no. 8, pp. 1044-1052, 2005.

[78] H. Kawano, S. Komaba, T. Kanamori, and Y. Kaneda, "A new therapy for highly effective tumor eradication using HVJ-E combined with chemotherapy," BMC Medicine, vol. 5, article 28, 2007.

[79] J. L. Ziegler, "Spontaneous remission in Burkitt's lymphoma," National Cancer Institute Monographs, vol. 44, pp. 61-65, 1976.

[80] T. Asada, "Treatment of human cancer with mumps virus," Cancer, vol. 34, no. 6, pp. 1907-1928, 1974.

[81] E. Kelly and S. J. Russell, "History of oncolytic viruses: genesis to genetic engineering," Molecular Therapy, vol. 15, no. 4, pp. 651659, 2007.

[82] J. J. Davis and B. Fang, "Oncolytic virotherapy for cancer treatment: challenges and solutions," Journal of Gene Medicine, vol. 7, no. 11, pp. 1380-1389, 2005.

[83] D. H. Kirn and S. H. Thorne, "Targeted and armed oncolytic poxviruses: a novel multi-mechanistic therapeutic class for cancer," Nature Reviews Cancer, vol. 9, no. 1, pp. 64-71, 2009.

[84] T.-C. Liu and D. Kirn, "Gene therapy progress and prospects cancer: oncolytic viruses," Gene Therapy, vol. 15, no. 12, pp. 877884, 2008.

[85] P. Msaouel, I. D. Iankov, C. Allen et al., "Engineered measles virus as a novel oncolytic therapy against prostate cancer," Prostate, vol. 69, no. 1, pp. 82-91, 2009.

[86] M. Kurooka and Y. Kaneda, "Inactivated Sendai virus particles eradicate tumors by inducing immune responses through blocking regulatory T cells," Cancer Research, vol. 67, no. 1, pp. 227-236, 2007. 
[87] T. Matsushima-Miyagi, K. Hatano, M. Nomura et al., "TRAIL and Noxa are selectively upregulated in prostate cancer cells downstream of the RIG-I/MAVS signaling pathway by nonreplicating Sendai virus particles," Clinical Cancer Research, vol. 18, no. 22, pp. 6271-6283, 2012.

[88] C. A. Piccirillo and E. M. Shevach, "Cutting edge: control of $\mathrm{CD}^{+} \mathrm{T}$ cell activation by $\mathrm{CD} 4^{+} \mathrm{CD} 25^{+}$immunoregulatory cells," Journal of Immunology, vol. 167, no. 3, pp. 1137-1140, 2001.

[89] S. Sakaguchi, "Naturally arising Foxp3-expressing CD $25^{+} \mathrm{CD} 4^{+}$ regulatory $\mathrm{T}$ cells in immunological tolerance to self and nonself," Nature Immunology, vol. 6, no. 4, pp. 345-352, 2005.

[90] T. Sasada, M. Kimura, Y. Yoshida, M. Kanai, and A. Takabayashi, " $\mathrm{CD} 4{ }^{+} \mathrm{CD} 25^{+}$regulatory $\mathrm{T}$ cells in patients with gastrointestinal malignancies: possible involvement of regulatory $\mathrm{T}$ cells in disease progression," Cancer, vol. 98, no. 5, pp. 1089-1099, 2003.

[91] J. M. Lund, L. Alexopoulou, A. Sato et al., "Recognition of single-stranded RNA viruses by toll-like receptor 7," Proceedings of the National Academy of Sciences of the United States of America, vol. 101, no. 15, pp. 5598-5603, 2004.

[92] K. Triantafilou, G. Orthopoulos, E. Vakakis et al., "Human cardiac inflammatory responses triggered by Coxsackie B viruses are mainly toll-like receptor (TLR) 8-dependent," Cellular Microbiology, vol. 7, no. 8, pp. 1117-1126, 2005.

[93] H. Kato, O. Takeuchi, S. Sato et al., "Differential roles of MDA5 and RIG-I helicases in the recognition of RNA viruses," Nature, vol. 441, no. 1, pp. 101-105, 2006.

[94] H. Suzuki, M. Kurooka, Y. Hiroaki, Y. Fujiyoshi, and Y. Kaneda, "Sendai virus F glycoprotein induces IL-6 production in dendritic cells in a fusion-independent manner," FEBS Letters, vol. 582, no. 9, pp. 1325-1329, 2008.

[95] A. Fujihara, M. Kurooka, T. Miki, and Y. Kaneda, "Intratumoral injection of inactivated Sendai virus particles elicits strong antitumor activity by enhancing local CXCL10 expression and systemic NK cell activation," Cancer Immunology, Immunotherapy, vol. 57, no. 1, pp. 73-84, 2008.

[96] Y. Kawaguchi, Y. Miyamoto, T. Inoue, and Y. Kaneda, "Efficient eradication of hormone-resistant human prostate cancers by inactivated Sendai virus particle," International Journal of Cancer, vol. 124, no. 10, pp. 2478-2487, 2009.

[97] R. B. Seth, L. Sun, C.-K. Ea, and Z. J. Chen, "Identification and characterization of MAVS, a mitochondrial antiviral signaling protein that activates NF- $\kappa$ B and IRF3," Cell, vol. 122, no. 5, pp. 669-682, 2005.

[98] P. A. Holoch and T. S. Griffith, "TNF-related apoptosis-inducing ligand (TRAIL): a new path to anti-cancer therapies," European Journal of Pharmacology, vol. 625, no. 1-3, pp. 63-72, 2009.

[99] C. Ploner, R. Kofler, and A. Villunger, "Noxa: at the tip of the balance between life and death," Oncogene, vol. 27, supplement 1, pp. S84-S92, 2008.

[100] P. Eitz Ferrer, S. Potthoff, S. Kirschnek et al., "Induction of Noxa-mediated apoptosis by modified vaccinia virus Ankara depends on viral recognition by cytosolic helicases, leading to IRF-3/IFN- $\beta$-dependent induction of pro-apoptotic Noxa," PLoS Pathogens, vol. 7, no. 6, Article ID e1002083, 2011.

[101] R. Romieu-Mourez, M. Solis, A. Nardin et al., "Distinct roles for IFN regulatory factor (IRF)-3 and IRF-7 in the activation of antitumor properties of human macrophages," Cancer Research, vol. 66, no. 21, pp. 10576-10585, 2006.

[102] M. T. Valentine, P. M. Fordyce, and S. M. Block, "Eg5 steps it up!", Cell Division, vol. 1, article 31, 2006.
[103] A. Blangy, H. A. Lane, P. d'Hérin, M. Harper, M. Kress, and E. A. Nigg, "Phosphorylation by $\mathrm{p} 34 \mathrm{cdc} 2$ regulates spindle association of human Eg5, a kinesin-related motor essential for bipolar spindle formation in vivo," Cell, vol. 83, no. 7, pp. 11591169, 1995.

[104] M. Matsuda, T. Yamamoto, A. Matsumura, and Y. Kaneda, "Highly efficient eradication of intracranial glioblastoma using Eg5 siRNA combined with HVJ envelope," Gene Therapy, vol. 16, no. 12, pp. 1465-1476, 2009.

[105] M. Matsuda, K. Nimura, T. Shimbo et al., "Immunogene therapy using immunomodulating HVJ-E vector augments anti-tumor effects in murine malignant glioma," Journal of Neuro-Oncology, vol. 103, no. 1, pp. 19-31, 2011.

[106] E. Villar and I. M. Barroso, "Role of sialic acid-containing molecules in paramyxovirus entry into the host cell: a minireview," Glycoconjugate Journal, vol. 23, no. 1-2, pp. 5-17, 2006.

[107] M. Nomura, T. Shimbo, Y. Miyamoto, M. Fukuzawa, and Y. Kaneda, "13-Cis retinoic acid can enhance the antitumor activity of non-replicating Sendai virus particle against neuroblastoma," Cancer Science, vol. 104, no. 2, pp. 238-244, 2013.

[108] T. Shimbo, M. Kawachi, K. Saga et al., "Development of a transferrin receptor-targeting HVJ-E vector," Biochemical and Biophysical Research Communications, vol. 364, no. 3, pp. 423428, 2007.

[109] K. Saga, K. Tamai, M. Kawachi et al., "Functional modification of Sendai virus by siRNA," Journal of Biotechnology, vol. 133, no. 3, pp. 386-394, 2008.

[110] E. N. Benveniste, "Cytokine actions in the central nervous system," Cytokine and Growth Factor Reviews, vol. 9, no. 3-4, pp. 259-275, 1998.

[111] M. P. Colombo and G. Trinchieri, "Interleukin-12 in anti-tumor immunity and immunotherapy," Cytokine and Growth Factor Reviews, vol. 13, no. 2, pp. 155-168, 2002.

[112] W. T. Watford, M. Moriguchi, A. Morinobu, and J. J. O'Shea, "The biology of IL-12: coordinating innate and adaptive immune responses," Cytokine and Growth Factor Reviews, vol. 14, no. 5, pp. 361-368, 2003.

[113] K. Saga, K. Tamai, T. Yamazaki, and Y. Kaneda, "Systemic administration of a novel immune-stimulatory pseudovirion suppresses lung metastatic melanoma by regionally enhancing IFN- $\gamma$ production," Clinical Cancer Research, vol. 19, no. 3, pp. 668-679, 2013.

[114] C. E. Meacham and S. J. Morrison, "Tumour heterogeneity and cancer cell plasticity," Nature, vol. 501, pp. 328-337, 2013. 

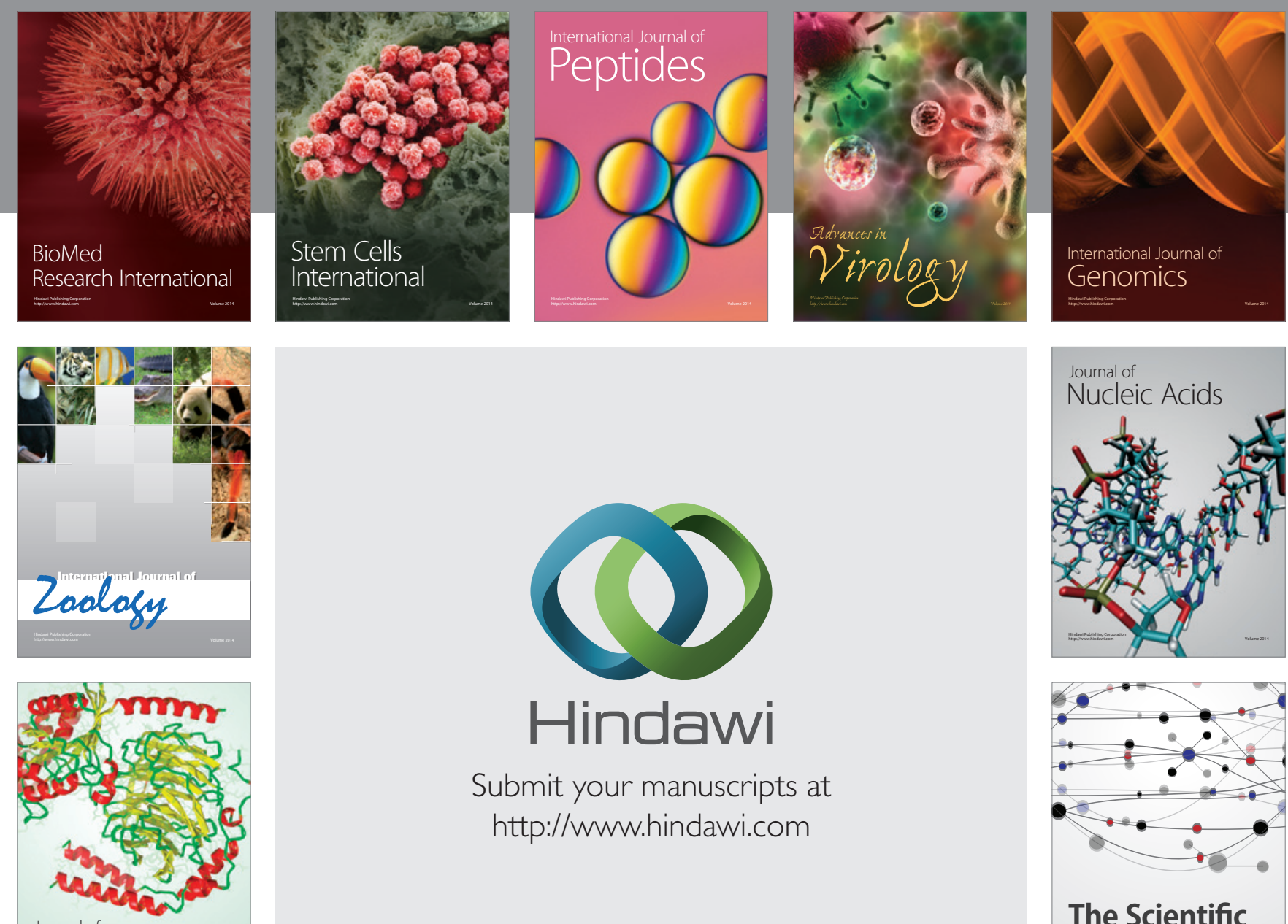

Submit your manuscripts at

http://www.hindawi.com

Journal of
Signal Transduction
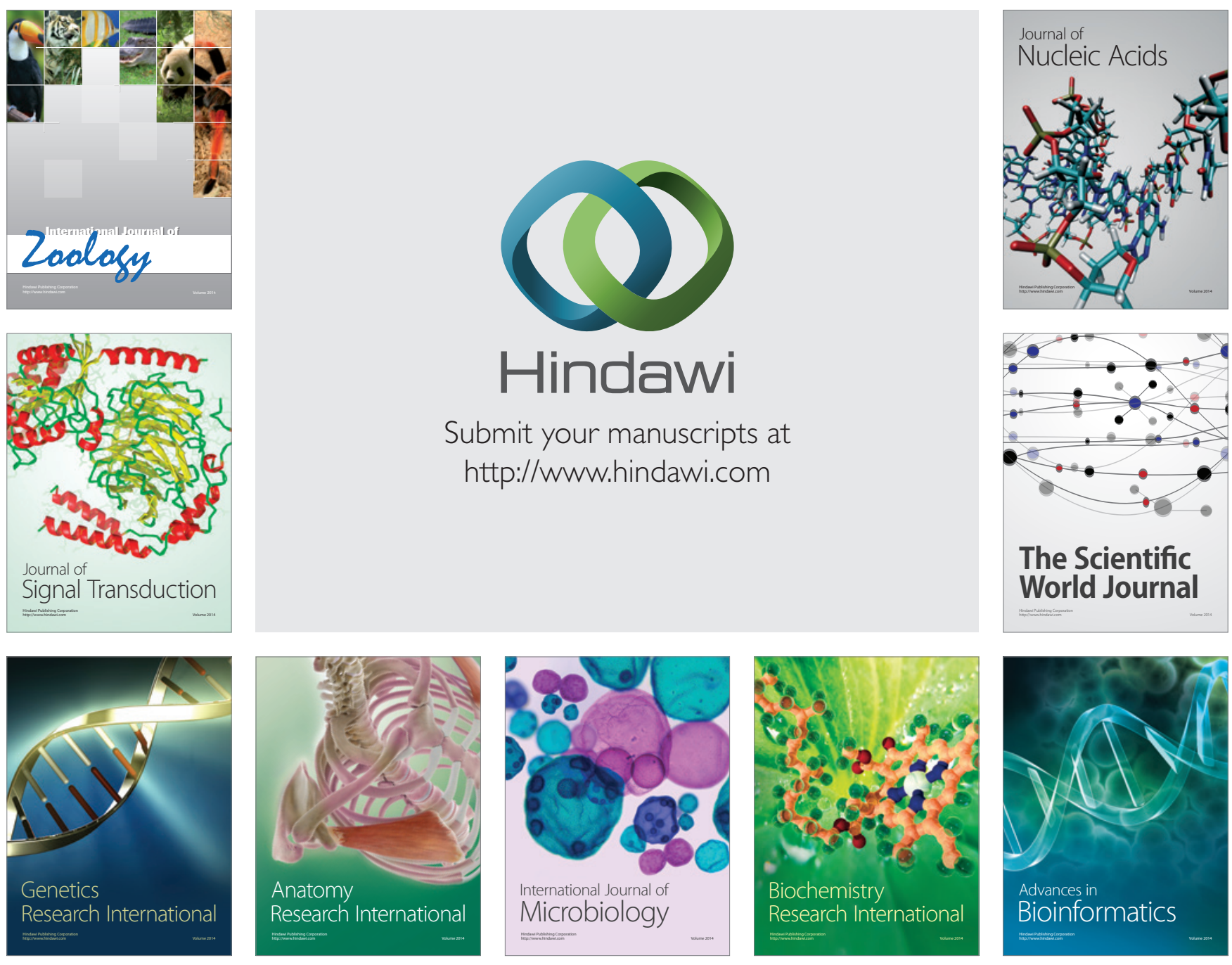

The Scientific World Journal
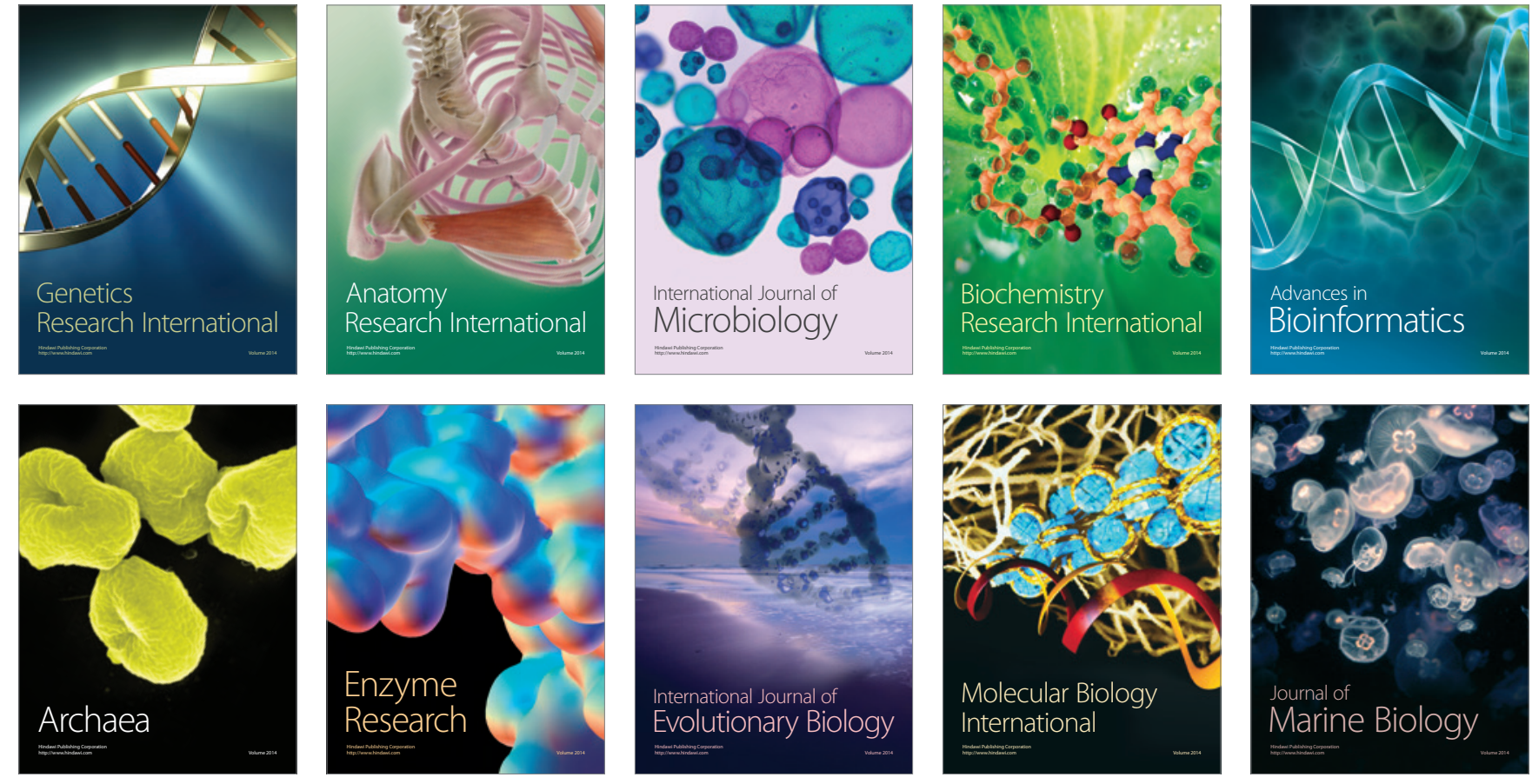